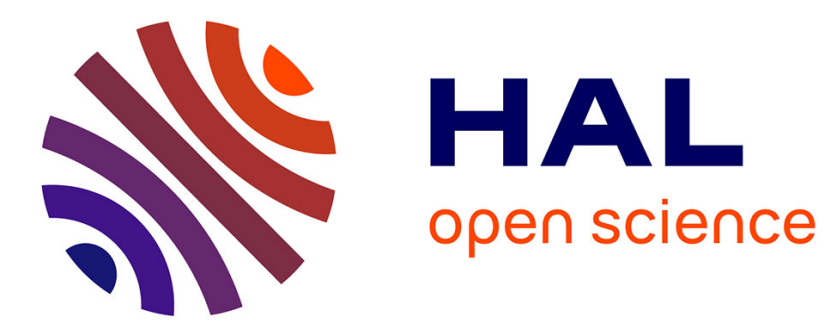

\title{
Transitions to chaos in the wake of an axisymmetric bluff body
}

\author{
Yannick Bury, Thierry Jardin
}

\section{To cite this version:}

Yannick Bury, Thierry Jardin. Transitions to chaos in the wake of an axisymmetric bluff body. Physics Letters A, 2012, vol. 376, pp. 3219-3222. 10.1016/j.physleta.2012.09.011 . hal-00781680

\section{HAL Id: hal-00781680 \\ https://hal.science/hal-00781680}

Submitted on 28 Jan 2013

HAL is a multi-disciplinary open access archive for the deposit and dissemination of scientific research documents, whether they are published or not. The documents may come from teaching and research institutions in France or abroad, or from public or private research centers.
L'archive ouverte pluridisciplinaire HAL, est destinée au dépôt et à la diffusion de documents scientifiques de niveau recherche, publiés ou non, émanant des établissements d'enseignement et de recherche français ou étrangers, des laboratoires publics ou privés. 


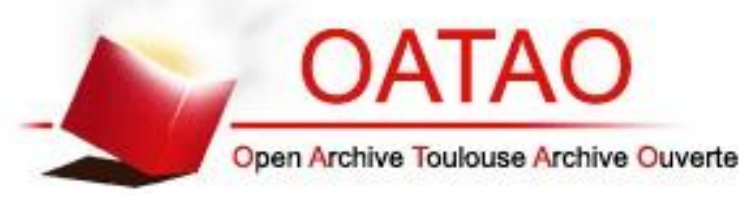

\section{Open Archive Toulouse Archive Ouverte (OATAO)}

OATAO is an open access repository that collects the work of Toulouse researchers and makes it freely available over the web where possible.

This is an author-deposited version published in: http://oatao.univ-toulouse.fr/ Eprints ID: 6503

To link to this article: DOI: 10.1016/j.physleta.2012.09.011

URL: http://dx.doi.org/10.1016/j.physleta.2012.09.011

To cite this version: Bury, Yannick and Jardin, Thierry Transitions to chaos in the wake of an axisymmetric bluff body. (2012) Physics Letters A, vol. 376 (n 45). pp. 3219-3222. ISSN 0375-9601 


\title{
Transitions to chaos in the wake of an axisymmetric bluff body
}

\author{
Yannick Bury, Thierry Jardin \\ Université de Toulouse, ISAE, 10, avenue Edouard Belin, 31055 Toulouse, France
}

\begin{abstract}
This letter aims at understanding the dynamical process that leads to the onset of chaos in the flow past a blunt-based axisymmetric bluff body. On the basis of direct numerical simulations, conducted for Reynolds numbers ranging from 100 to 900 , we show that the flow undergoes multiple transitions, successively giving rise to the $\mathrm{SS}_{,} \mathrm{RSP}_{a}, \mathrm{RSP}_{b}, \mathrm{RSP}_{c}$ and RSB wake states. In particular, the $\mathrm{RSP}_{c}$ state, revealed in this work via long-term computations, is characterized by intermittent vortex stretching denoting the onset of chaos before the symmetry breaking and the occurence of the RSB state.
\end{abstract}

Keywords: flow instabilities, chaos, wake, bluff body

\section{Introduction}

The thorough analysis of bluff body wakes is essentially twofold. First, it advances the knowledge of fundamental mechanisms driving the destabilization of massively separated flows, i.e. the transition between dominant flow instabilities. Second, as an ultimate goal, it serves the definition of optimal flow control strategies through the manipulation of such flow instabilities. As such, it has been proven, for low Reynolds number flows, that forcing the flow at specific forcing frequencies/wavelengths can drastically modify the wake topology and the drag experienced by the bluff body $[1,2,3]$. Moreover it is reasonable to assume that instabilities observed at low Reynolds numbers still persist at much higher Reynolds numbers. An evident but still fascinating example is the clear occurence of the von Kármán vortex shedding in the wake of geological obstacles, for Reynolds numbers $\mathcal{O}\left(10^{9}\right)$. Therefore one can expect a flow control strategy, primarily optimized at low Reynolds number, to be efficient at higher Reynolds number. In this sense the effort to enhance knowledge of the flow destabilization towards chaotic states is a prerequisite for the potential definition of optimal flow control strategies at higher Reynolds numbers. In this letter, the wake instabilities behind a blunt-based axisymmetric bluff body at various Reynolds numbers, ranging from 100 to 900, are analysed. Wakes of axisymmetric bluff bodies at low Reynolds number have gained interest due to the variety of instabilities that develop as the Reynolds number is increased, eventually leading to chaotic state. $\mathrm{Nu}-$ merous studies focused on spheres, revealing transitions of the wake structure from 1) an axisymmetric steady state

Email address: yannick.bury@isae.fr, thierry.jardin@isae.fr (Yannick Bury, Thierry Jardin) (corresponding to the base flow) to 2) a reflectional symmetry steady state (SS), 3) a reflectional symmetry preserving (RSP) oscillatory state, and 4) a reflectional symmetry breaking (RSB) oscillatory state [4, 5]. Such transitions and resulting wake structures exhibit similarities with wakes that develop downstream blunt-based axisymmetric bluff bodies [6, 7]. However this geometry is still poorly documented despite its practical interest and a lack of comprehension subsists regarding the scenario that leads to a chaotic state.

In order to decipher this scenario, we have conducted 3D direct numerical simulations of the flow past a bluntbased axisymmetric bluff body of length-to-diameter ratio $L / D=7$ for Reynolds numbers $100<R e<900$, spanning the transitions up to chaos.

\section{Numerical methods}

The three-dimensional time-dependent incompressible Navier-Stokes equations around a blunt-based axisymmetric bluff body of diameter $D$ and length $L$ are directly solved using an Eulerian finite volume method. The Reynolds number $R e$, based on $D$ and on the free stream velocity $U_{\infty}$, is varied from 100 to 900 . Assuming incompressible viscous flow, the equations read:

$$
\begin{aligned}
& \vec{\nabla} \cdot \vec{v}=0 \\
& \frac{\partial \vec{v}}{\partial t}+(\vec{v} \cdot \vec{\nabla}) \vec{v}=-\frac{1}{\rho} \vec{\nabla} p+\nu \nabla^{2} \vec{v}
\end{aligned}
$$

Here $\vec{v}$ is the velocity, $p$ the pressure, $\rho$ and $\nu$ the fluid density and kinematic viscosity respectively.

The geometry consists of a 4:1 semi-elliptic nose and a cylindrical aft section. It is enclosed in a cylindrical computational domain of diameter $20 \mathrm{D}$ and length $55 \mathrm{D}$ 

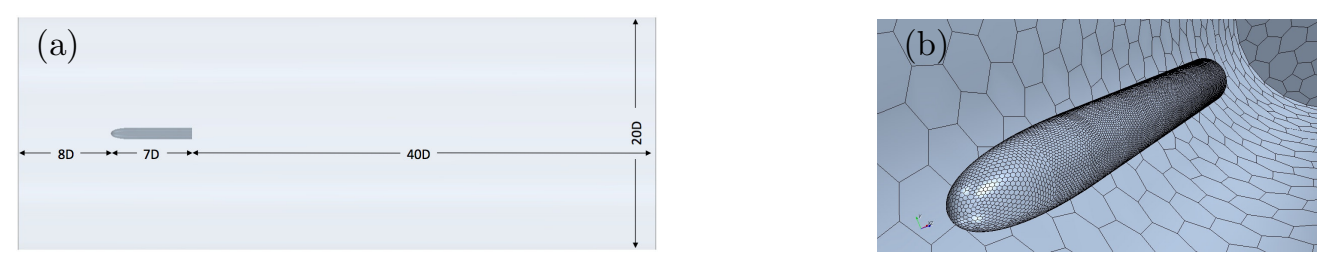

Figure 1: Computational domain (a) and illustration of the surfacic polyhedral mesh (b).

aligned with the free stream direction $\vec{x}$ (figure $1(\mathrm{a})$ ). The coordinates' origin is located at the nose tip of the body, corresponding to the stagnation point. The inlet boundary is located at $x / D=-8$ and is subjected to a velocity Dirichlet condition. A similar condition is imposed on the tubular surface of the computational domain. A zero diffusion flux condition is prescribed at the outlet boundary located at $x / D=47$. The surface of the blunt-based axisymmetric bluff body is modelled as non-slip surface. The domain is composed of $2.3 \times 10^{6}$ polyhedral cells (figure $1(\mathrm{~b}))$.

The spatial and temporal discretizations are achieved using second-order upwind schemes and second-order implicit time-stepping method respectively. The pressurevelocity coupling is obtained using a SIMPLE algorithm. Time step is fixed in order to satisfy the CFL condition (Courant Number close to unity) for each Reynolds number.

\section{Results}

Figure 2 illustrates a sequence of the flow topology obtained for $R e$ numbers ranging from 100 to 450, in terms of iso-surfaces of normalized $\lambda_{2}^{+}$[8] (normalization is based on the minimum value of $\lambda_{2}$ for each Reynolds number considered).

At $R e=100$, figure 2 depicts an opened torus of revolution at the base of the body. The associated recirculation region is axisymmetric. Between $R e=100$ and $R e=400$ the torus radially expands towards the external flow and progressively stretches along the streamwise direction. This scenario results from the concomitant influence of strong shear and suction effects on the torus. While the low-pressure region maintains the opened torus close to the bluff body base, the external flow tends to stretch the periphery of the opened torus.

As the Reynolds number is increased up to $R e \approx 450$ the torus progressively shifts away from the bluff body axis of revolution. Hence, the upper part of the torus is subjected to stronger shear than the lower part, such that it distorts into a pelvis-like structure. This is associated with the loss of axisymmetry of the torus, corresponding to the destabilization of the wake towards the reflectional symmetry steady state (SS). At this stage only a single streamwise planar symmetry is retained whose azimuthal position fixes randomly.
The streamwise vorticity fields provide additional information on the topology of the flow where the asymmetry is reflected as a 'double-threaded' wake composed of two primary vorticity lobes distributed on both sides of the reflectional symmetry plane (figure 3 ). Secondary and ternary vorticity lobes of alternate vorticity signs are also observed in the near wake and on the body aft surface respectively. As the Reynolds number is further increased, the tails of the primary vorticity lobes elongate and shift away from the axis of revolution of the bluff body (see also [7]), in accordance with the pelvis-like structure displacement and distortion under free stream-induced shear stresses. At this stage the pelvis-like structure is subjected to increasing shear stresses in conjunction with its displacement towards the external flow.

Eventually as the Reynolds number reaches values close to 590, the structure can no more sustain such stresses and is partially torn. This leads to the formation of unsteady hairpin structures periodically shed in the wake of the blunt-based axisymmetric bluff body (figure 4(a: right)). The reflectional symmetry is still preserved, denoting the transition from the SS state to the RSP oscillatory state. Figure 4(a: left) depicts iso-surfaces of streamwise vorticity $\omega^{+}$at $R e=600$. It displays regularly spaced vorticity lobes shed at a non-dimensional frequency $S t_{a}=f_{a} D / U_{\infty} \approx 0.12$. At this stage the flow exhibits a unique dominant frequency, revealed in figure 5 (a) by a single fundamental peak (and its first harmonic) on the FFT of the drag coefficient history. The fundamental peak amplitude increases as $R e$ is varied from 590 to 680 . Hereafter this single frequency RSP state will be denoted $\mathrm{RSP}_{a}$.

Beyond $R e=690$ a second fundamental peak (and its first harmonic) arises at $S t_{b} \approx 0.02$ (figure $5(\mathrm{~b})$ ), denoting the occurence of low frequency vorticity bursts that promote the transient waving of the primary lobes and of the associated hairpin structures in the reflectional symmetry plane (figure 4(b)). The occurence of this second fundamental peak is accompanied with beating frequencies $S t_{a}-S t_{b}$ and $S t_{a}+S t_{b}$ that result from its coupling with the first fundamental peak. This double frequency RSP state will be denoted $\mathrm{RSP}_{b}$. Note that the second to first fundamental peaks ratio increases with $R e$, which indicates that drag fluctuations, initially driven by the shedding of hairpin vortices at $S t_{a}$ are progressively dominated by the vorticity bursts and the associated transient waving 
(a)
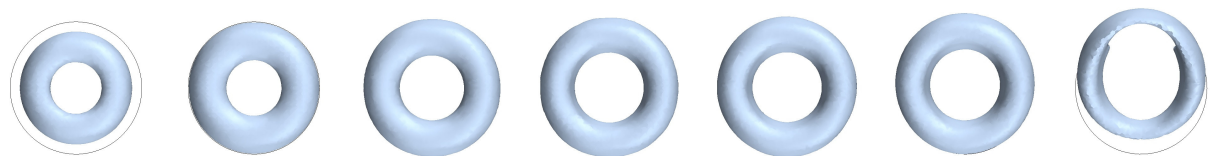

(b)
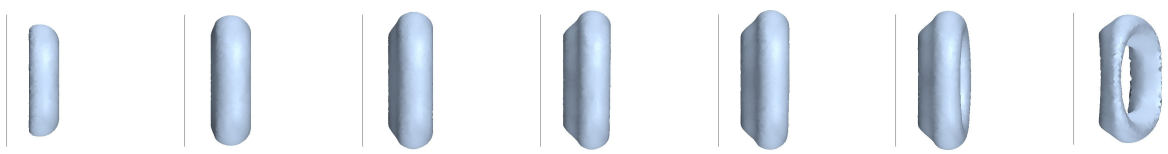

(c)
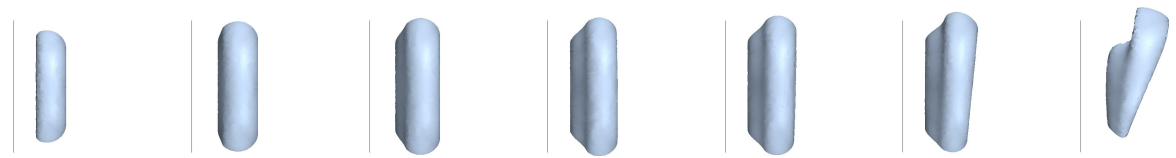

$R e=100$

200

300

400

420

430

450

Figure 2: Iso-surfaces of $\lambda_{2}^{+}=-0.02$ obtained for Re numbers ranging from 100 to 450 . Solid line depicts the base of the bluff body. (a), (b) and (c) correspond to rear, lower and side views respectively.
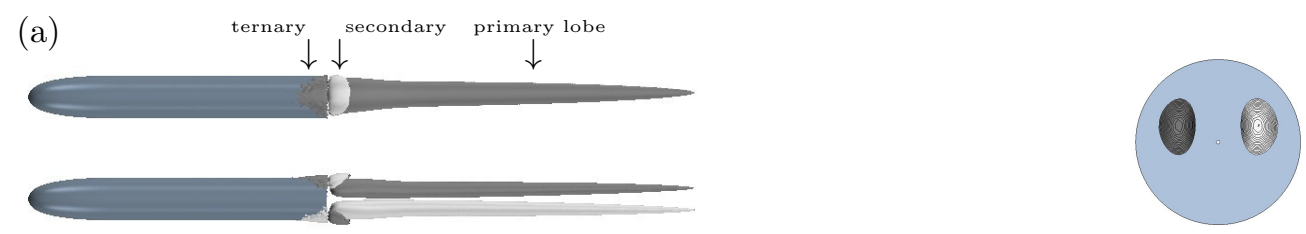

(b)
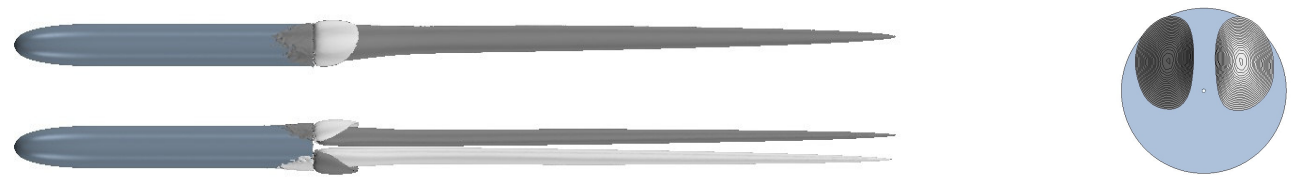

Figure 3: Normalized streamwise vorticity $\omega^{+}=\omega D / U_{\infty}$ obtained for Re numbers 450 (a) and 550 (b). Left: lower and side views of iso-surfaces $\omega^{+}= \pm 0.05$. Right: rear view of isolines $\left|\omega^{+}\right| \in[0.05 ; 0.15]$ obtained at $x / D=14$ (dark: positive, light: negative).

(a)

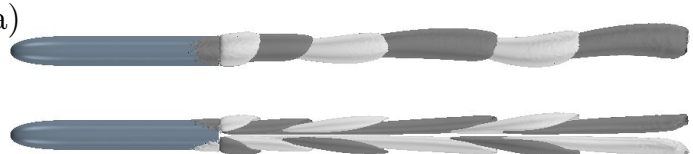

(b)

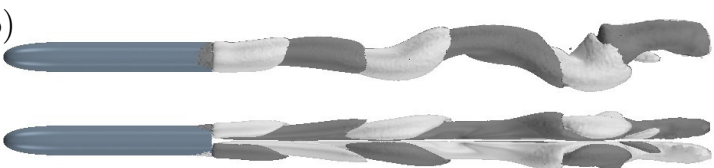

(c)
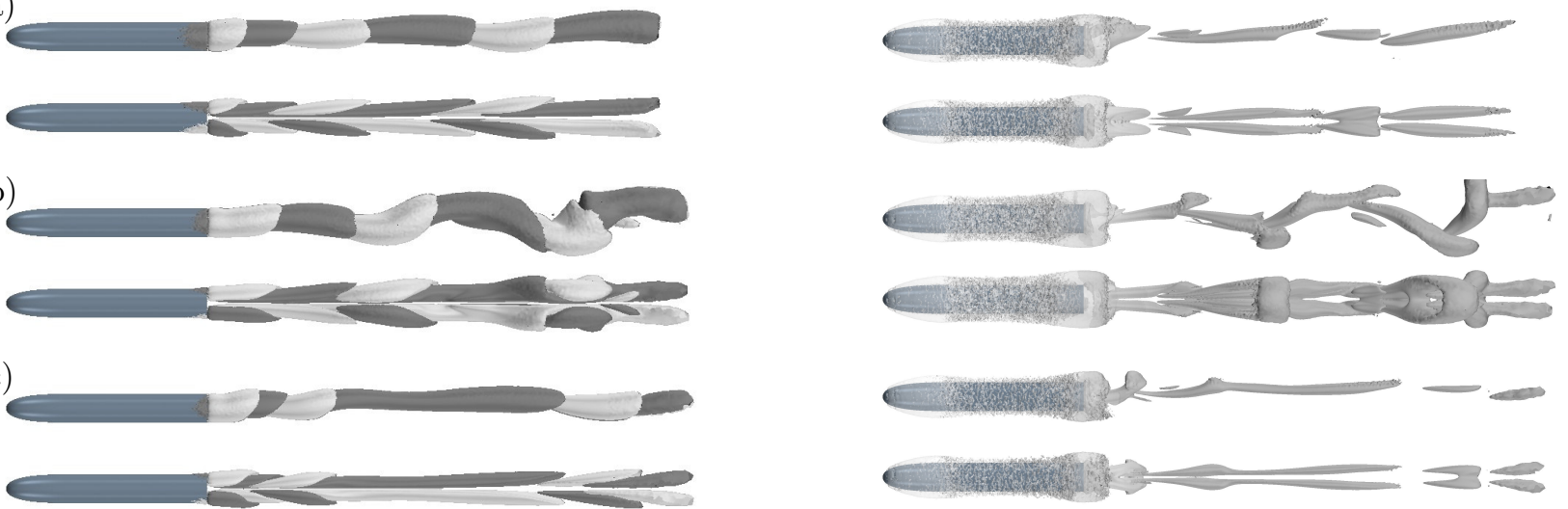

Figure 4: Lower and side views of the flow structure obtained for Re numbers 600 (a), 700 (b) and 800 (c). Left: iso-surfaces $\omega^{+}= \pm 0.05$ (dark: positive, light: negative). Right: iso-surfaces $\lambda_{2}^{+}<0$. 
(a)

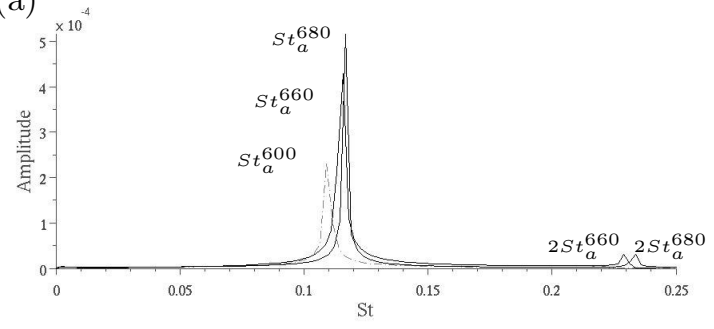

(c)

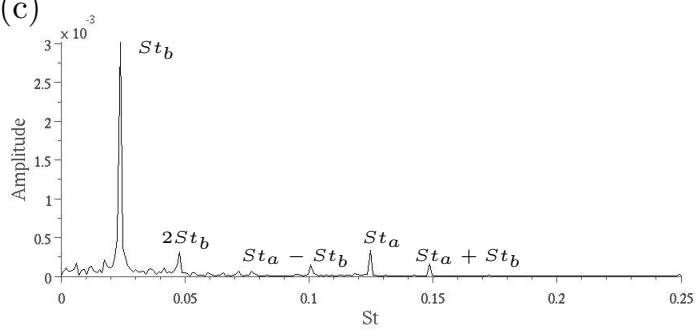

(b)

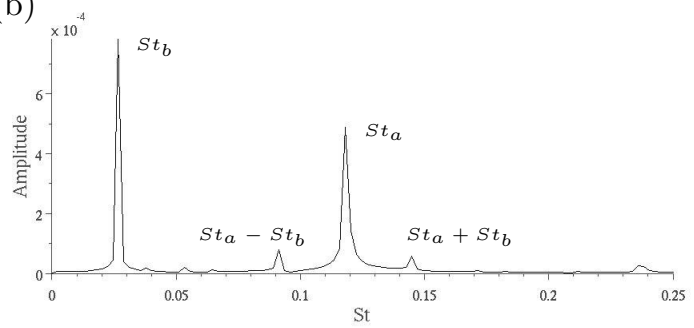

(d)

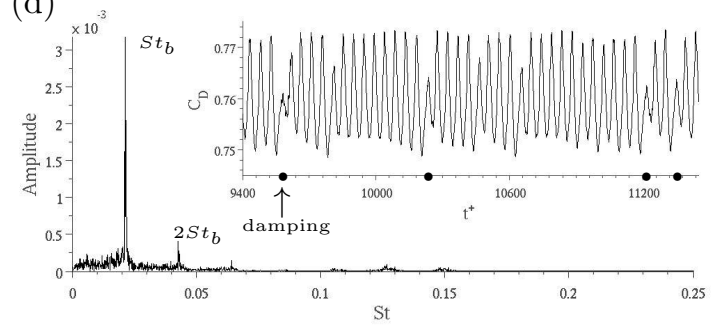

Figure 5: FFT of the drag coefficient history obtained for Re numbers 600 (dotted line), 660 (dashed line), 680 (plain line) (a), 700 (b), 750 (c) and 800 (inset picture depicts the drag coefficient history) (d).

of the wake at $S t_{b}$.

Beyond $R e=750$ a broadband noise appears on the FFT spectrum of the drag coefficient history (figure 5(c, d)), revealing the transition to chaos potentially induced by the occurence of a third fundamental frequency, following the Ruelle-Takens-Newhouse scenario [9]. At this stage the analysis of this chaotic state deserves a deeper attention. To this avail a 12000 time units computation has been conducted at $R e=800$, which roughly corresponds to the upper bound of the $\operatorname{RSP}_{c}$ state. Here the time unit is defined as $t^{+}=t U_{\infty} / D$. This long term computation allows both a better resolution of the broadband noise and the capture of very low frequency instabilities. The prominent feature of the $\mathrm{RSP}_{c}$ state is the transient stretching of the hairpin vortices in the streamwise direction (figure $4(\mathrm{c})$ ), whose associated vorticity lobes remind the 'doublethreaded' wake observed for the SS state. This could suggest a transient restabilization of the wake. It results in the damping of the drag oscillations, clearly observed on the drag coefficient history illustrated on the inset picture of figure $5(\mathrm{~d})$. It is remarkable that this damping occurs intermittently. In that sense, this phenomenon is a marker of the chaotic state of the flow associated with the potential emergence of a third instability.

The further increase of the Reynolds number finally breaks the reflectional symmetry and triggers the RSB state. For $R e=900$, phases of 'apparent' reflectional symmetry (figure 6(a)) whose flow topology ressembles that of the $R S P_{b}$ state are randomly interrupted by dramatic changes of the symmetry plane azimuthal position (figure $6(\mathrm{c})$ ). Such changes, also characterized by strong intermittent oscillations of the lateral loads (figure 7(b)), occur immediately after a transient phase of wake twisting (figure 6(b)). In addition the long-term, 9000 time units computation achieved for $R e=900$ reveals the persistence of the intermittent damping of the drag coefficient (figure $7(\mathrm{a}))$ and of the associated vorticity lobes stretching (figure $6(\mathrm{~d})$ ), previously observed at $R e=800$.

\section{Conclusion}

The transition to chaos of the flow past a blunt-based axisymmetric bluff body can thus be deciphered as a succession of bifurcations associated with distinct wake patterns. In particular the dynamics of the recirculation region from an axisymmetric torus (base flow) to a pelvis-like structure (SS state) subsequently leads to the oscillatory RSP state. This RSP state can be subdivided into three substates, whether the flow unsteadiness is characterized by a single fundamental frequency $\left(\mathrm{RSP}_{a}\right)$, two distinct fundamental frequencies $\left(\mathrm{RSP}_{b}\right)$ or a chaotic dynamics $\left(\mathrm{RSP}_{c}\right)$. The latter exhibits flow intermittencies associated with the transient stretching of the hairpin structures and the damping of the drag oscillations. The scenario revealed in this work shows strong similarities with the RuelleTakens-Newhouse scenario, although the precise identification of the 'chaos triggering' frequency is still unclear and requires deeper investigation. In addition these intermittencies still persist beyond the bifurcation towards the RSB state whose wake topology consists in a succession of phases of 'apparent' reflectional symmetry, wake twisting and dramatic reorientation of the 'apparent' reflectional symmetry. 
(a)

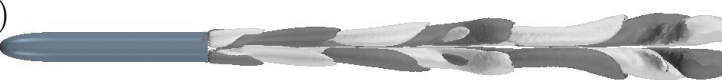

(c)

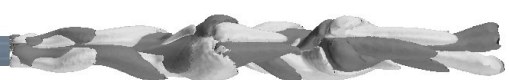

(b)

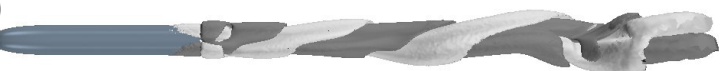

(d)

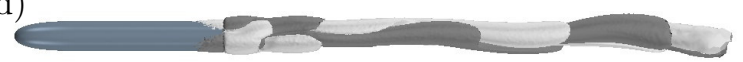

Figure 6: Iso-surfaces $\omega^{+}= \pm 0.05$ (dark: positive, light: negative) of the flow obtained for $R e=900$ at different phases of the RSB state. 'Apparent' reflectional symmetry (a), wake twisting (b), reorientation of the 'apparent' reflectional symmetry (c), vorticity lobes stretching (d).

(a)

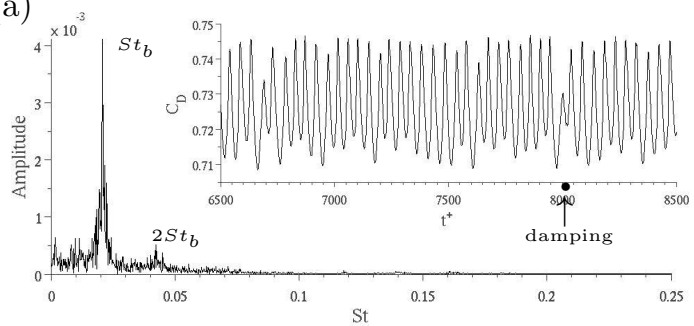

(b)

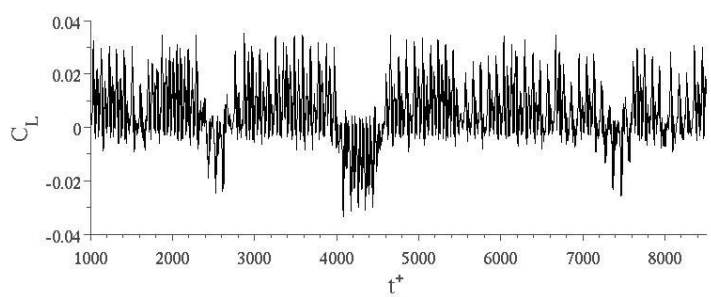

Figure 7: FFT of the drag coefficient history obtained for $R e=900$ (inset picture depicts the drag coefficient history) (a) and lateral force coefficient history (b).

\section{Acknowledgements}

The authors gratefully acknowledge the French Ministry of Defence and the DGA for supporting this work.

\section{References}

[1] J. Kim and H. Choi 2005, Distributed forcing of the flow over a circular cylinder. Phys. Fluids, 17:033103.

[2] H. Choi, W.P. Jeon and J. Kim 2008, Control of flow over a bluff body. Annu. Rev. Fluid Mech., 40:113-139.

[3] T. Jardin and Y. Bury 2012, Spectral and lagrangian analysis of the forced flow past a circular cylinder using pulsed tangential jets. J. Fluid Mech., 696:285-300.

[4] A. G. Tomboulides and S.A. Orszag 2000, Numerical investigation of transitional and weak turbulent flow past a sphere. $J$. Fluid Mech., 416:51-73.

[5] D. Fabre, F. Auguste and J. Magnaudet 2008, Bifurcations and symmetry breaking in the wake of axisymmetric bodies. Phys. Fluids, 20:051702.

[6] V. Schwarz, H. Bestek and H. Fasel 1994, Numerical simulation of nonlinear waves in the wake of an axisymmetric bluff body. In 25th AIAA Fluid Dynamics Conference, AIAA-94:2285.

[7] P. Bohorquez, E. Sanmiguel-Rojas, A. Sevilla, J.I. JimenezGonzalez and C. Martinez-Bazan 2011, Stability and dynamics of the laminar wake past a slender blunt-based axisymmetric body. J. Fluid Mech., 676:110-144.

[8] J. Jeong and F. Hussain 1995, On the identification of a vortex. J. Fluid Mech., 285:69-94.

[9] J.P. Eckmann 1981, Roads to turbulence in dissipative dynamical systems. Rev. Mod. Phys., 53:643-654. 